\title{
Development of a Novel Embedded Relay Lens Microscopic Hyperspectral Imaging System for Cancer Diagnosis: Use of the Mice with Oral Cancer to Be the Example
}

\author{
Yao-Fang Hsieh, ${ }^{1}$ Mang Ou-Yang, ${ }^{2}$ Jeng-Ren Duann, ${ }^{3,4}$ Jin-Chern Chiou, ${ }^{2,4}$ \\ Nai-Wen Chang, ${ }^{5}$ Chia-Ing Jan, ${ }^{6,7,8}$ Ming-Hsui Tsai, ${ }^{9,}{ }^{10}$ Shuen-De Wu, ${ }^{11}$ \\ Yung-Jiun Lin, ${ }^{4}$ and Cheng-Chung Lee ${ }^{1}$
}

\begin{abstract}
${ }^{1}$ Department of Optics and Photonics, National Central University, 300 Jhongda Road, Taoyuan, Chungli 32001, Taiwan
${ }^{2}$ Department of Electrical and Computer Engineering, National Chiao Tung University, 1001 University Road, Hsinchu 30010, Taiwan

${ }^{3}$ Graduate Institute of Clinical Medical Science, China Medical University, 91 Hsueh-Shih Road, Taichung 40402, Taiwan

${ }^{4}$ Biomedical Engineering Research and Development Center, China Medical University Hospital, 2 Yuh-Der Road,

Taichung 40447, Taiwan

${ }^{5}$ Department of Biochemistry, College of Medicine, China Medical University, 91 Hsueh-Shih Road, Taichung 40402, Taiwan

${ }^{6}$ Department of Pathology, China Medical University Hospital, 2 Yuh-Der Road, Taichung 40447, Taiwan

${ }^{7}$ Department of Dentistry, National Yang-Ming University, 155 Linong Street Section 2, Taipei 112, Taiwan

${ }^{8}$ Department of Pathology, China Medical University Beigang Hospital, 123 Xinde Road, Yunlin 651, Taiwan

${ }^{9}$ Department of Otolaryngology, China Medical University, 91 Hsueh-Shih Road, Taichung 40402, Taiwan

${ }^{10}$ Department of Otolaryngology Head Neck Surgery, China Medical University Hospital, 2 Yuh-Der Road, Taichung 40447, Taiwan

${ }^{11}$ Department of Mechatronic Technology, National Taiwan Normal University, 162 Heping East Road Section 1, Taipei 106, Taiwan
\end{abstract}

Correspondence should be addressed to Mang Ou-Yang, oym@cc.nctu.edu.tw

Received 30 June 2012; Revised 6 October 2012; Accepted 20 October 2012

Academic Editor: Mohammed A. Gondal

Copyright ( $) 2012$ Yao-Fang Hsieh et al. This is an open access article distributed under the Creative Commons Attribution License, which permits unrestricted use, distribution, and reproduction in any medium, provided the original work is properly cited.

\begin{abstract}
This paper develops a novel embedded relay lens microscopic hyperspectral imaging system (ERL-MHSI) with high spectral resolution (nominal spectral resolution of $2.8 \mathrm{~nm}$ ) and spatial resolution $(30 \mu \mathrm{m} \times 10 \mu \mathrm{m})$ for cancer diagnosis. The ERL-MHSI system has transmittance and fluorescence mode. The transmittance can provide the morphological information for pathological diagnosis, and the fluorescence of cells or tissue can provide the characteristic signature for identification of normal and abnormal. In this work, the development of the ERL-MHSI system is discussed and the capability of the system is demonstrated by diagnosing early stage oral cancer of twenty mice in vitro. The best sensitivity for identifying normal cells and squamous cell carcinoma (SCC) was $100 \%$. The best specificity for identifying normal cells and SCC was $99 \%$. The best sensitivity for identifying normal cells and dysplasia was $99 \%$. The best specificity for identifying normal cells and dysplasia was $97 \%$. This work also utilizes fractal dimension to analyze the morphological information and find the significant different values between normal and SCC.
\end{abstract}

\section{Introduction}

The hyperspectral image (HSI) is capable of simultaneously presenting spectral and spatial information with high resolution. The spectral information provides the characteristic of objects, and the spatial information provides the morphological information of objects. The combination allows for spectral analysis of each pixel on the acquired image and assists statistical image analysis of the acquired image. Therefore, the HSI has been widely applied to many areas, such as remote sensing, digital archives, biomedical inspection, and so on [1]. In the biomedical inspection, the HSI is a useful modality in diagnostic medicine including applications for retinal image [2, 3], skin diagnosis [4-7], tumor microvasculature change, and cancer diagnosis [810]. Biological tissues have optical characteristics reflecting 
the chemical characteristics to provide information with regard to the health or disease of tissue. Because the cancer is the high mortality and morbidity disease, the physician hopes to find the characteristic of cancer in the early stage. The most accurate way to diagnose cancer relies on pathologist to study biopsy under the optical microscopic image. Although the optical microscope provides the direct image of the biopsy and is the most important instrument to research pathological change of cancer cell, the optical microscope still has the limitations. The interaction between light and the object changes the phase of the light wave and produces the interference effects. Also, the different experience and degree of subjectivity for identification borderline dysplastic cells among pathologists need to be considered. Therefore, the technique of combination of microscopic image and HSI has been developed to diagnose cancer [11]. The technique named microscopic hyperspectral image (MHSI) can record the morphological property of tissue and the spectral signals of each pixel of tissue image. The spectral information of MHSI always bases on the fluorescent signal. The fluorescence signal depends on the interaction of light with some components of cell. Proteins, enzymes (collagen, porphyrin), amino acid, and coenzymes (NADH, riboflavins) interact with the light of specific wavelength. The qualitative and quantitative differences of cell fluorophores were utilized to distinguish malignant from normal tissues [12-15]. The common useful fluorescence-based optical techniques are compared in Table 1. The Anwer's team used the morphologic image and fluorescent signal of MHSI to diagnose the cervical cancer [16]. They totally took 308 fibroblast cells to be the sample for analysis. The system identified normal cervical cells with a specificity of $95.8 \%$. As to low-grade precancerous cells and high-grade precancerous, the sensitivity was $66.7 \%$ and $93.5 \%$, respectively. The Matthew's team used the MHSI to diagnose the skin cancer of mouse, and the difference of spectral information between normal mouse and malignant mouse was obvious [17]. They used five mice to be the sample and got 116 hyperspectral images. Finally, they find that the optimized excitation wavelength of fluorescence was $420 \mathrm{~nm}$. The Hamed's team used ten resected stomach to be the sample and got 101 infrared hyperspectral images [18]. They showed 90\% specification. The Masood's team used the morphologic image of MHSI to classify the colon tissue and got $84 \%$ classification rate [19]. The MHSI system was preliminary successful applied to the cancer detection.

However, according to the principle of hyperspectral image, the MHSI system needed a scanning platform to scan the image and then acquire the entire hyperspectral image data. The previous researches [1, 8-12, 16-19] used the pushbroom structure to be the scanning mechanism of MHSI system. Figure 1(a) shows the structure of traditional pushbroom MHSI system. The system was enormous and complicated which needed larger space for usage. Because the pushbroom scanning mechanism was under the sample stage, the slightly vibration would affect the imaging quality. Hence, the stability and precision of the mechanism were very important. The driver of the pushbroom scanning mechanism utilized piezoelectric transducer (PZT) which was expensive. Also, when the objective power was changed, the moving distance of the PZT by per scan must be changed. This would cause more scanning time and inconvenience of usage. Because the structure was complexity, the optical axis of the pushbroom MHSI also was not easy to align. When the optical axis of system canot have good alignment, the quality and spectral information of the image were not good because of the optical aberration (e.g., color aberration). The color aberration was a very important parameter for the MHSI system, because it affected the fluorescence spectral information of cells. Besides, the off-axis aberration was the big problem of the pushbroom MHSI system especially in the high magnification of objective lens condition, because the entire system had no off-axis calibration. The off-axis aberration caused the serious image distortion. The distortion would affect the morphological information of the image. Hence, this paper proposes a novel embedded relay lens microscopic hyperspectral imaging (ERL-MHSI) system that used our previous design to be the scanning part [20]. The demonstrated diagrams of the proposed system are showed in Figure 1(b). The designed relay lens (RL) for scanning is put between the microscope and the hyperspectrometer (HS). The stepping motor (SM) is under the RL. The RL is particularly designed with symmetric infinite conjugate lenses for scanning and transferring images with optimal off-axis optical aberration (distortion $<0.02 \%$, field curvature $<0.2 \mu \mathrm{m}$ ). The mechanism of proposed system makes the objective plane (IMP1) and imaging plane (IMP2) on the same optical plane. When the objective lens changes the magnification, the image of object and the image on the slit of hyperspectrometer have the same magnification, the moving distance by per scan does not need to change. Hence, the novel system can optically change the scanning mechanism of nanometer-level resolution needed in a conventional MHSI system, which can only be accomplished by utilizing a PZT mechanism, that of micrometer-level resolution. The latter can be easily carried out by an ordinary SM, which dramatically reduces the cost of the proposed ERL-MHSI system. The entire volume of the proposed system $(70 \mathrm{~cm}(L) \times 55 \mathrm{~cm}(W) \times 80 \mathrm{~cm}(H))$ is smaller than the conventional system $(120 \mathrm{~cm}(L) \times$ $100 \mathrm{~cm}(W) \times 95 \mathrm{~cm}(H))$. A comparison of the ERL-MHSI system and pushbroom MHSI system is listed in Table 2.

According to the statistics of the American Cancer Society, approximately 40,250 new cases of oral and throat cancer are expected in the 2012. The oral cancer is the sixth common cancer and leads to about 570,000 deaths every year worldwide [21]. In the USA, the overall 5-year survival is $61 \%$ of all stages and decreases to $56 \%$ of the regional disease. The incidences rates of men are higher than women. Despite the advances in therapy, the 5-year survival rate has not obviously raised during the past two decades, because the therapy is more effective for patients in early stage, but, most patients appear to be the advanced stage for which therapy is less effective and brings worry in swallowing, talking, and face. Early detection of neoplastic changes is the best way to improve these events. Therefore, this paper demonstrates the capability of the proposed ERL-MHSI system by identifying the early stage oral cancer of mice. Twenty mice were utilized to be the biopsy samples. The fluorescence 


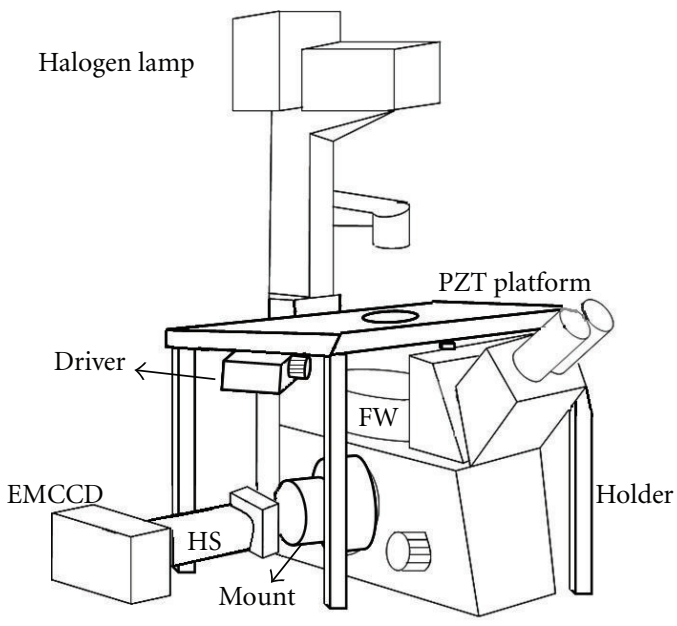

(a)

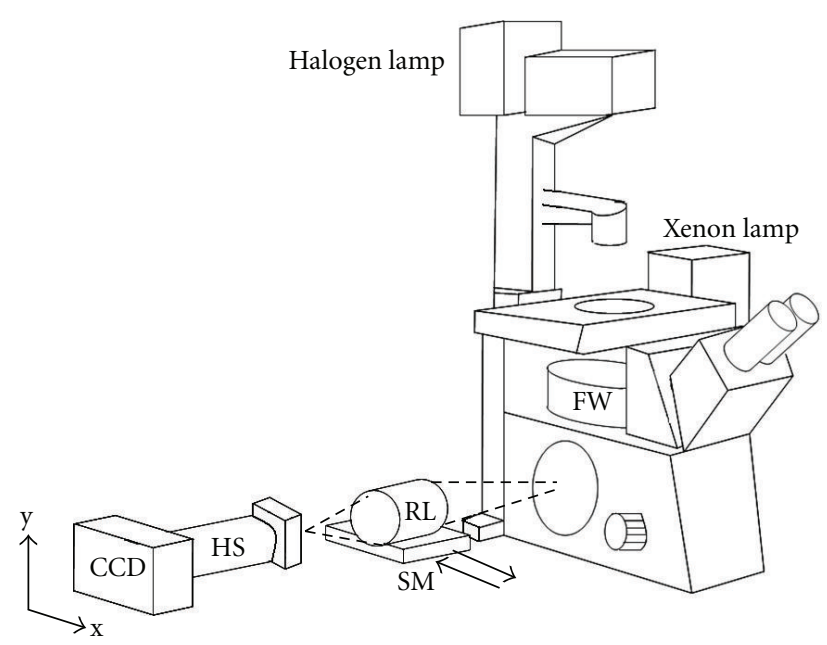

(b)

FIGURE 1: (a) The sketch of conventional MHSI system. (b) The sketch of proposed ERL-MHSI system.

TABLE 1: The comparison of four fluorescence based optical techniques.

\begin{tabular}{|c|c|c|c|c|}
\hline Optical technique & Hyperspectrum & Confocal & OCT & Raman \\
\hline Scanning range & Wide & Wide & Narrow & Narrow \\
\hline Scanning time & Median & Slow & Fast & Fast \\
\hline Spectral range & Wide & Wide & Narrow & Wide \\
\hline Spatial resolution & $\begin{array}{c}\text { By objective } \\
\text { (for } 20 \mathrm{x}, 0.5 \mu \mathrm{m} \text { ) }\end{array}$ & By objective & $10 \mu \mathrm{m}($ axial $)$ & By objective \\
\hline Depth scanning & No & Yes & Yes & No \\
\hline Main application & $\begin{array}{c}\text { Biomedical image, } \\
\text { Classification of biomolecule }\end{array}$ & Biomedical image & Biomedical image & Identification of biomolecule \\
\hline
\end{tabular}

The OCT represents the Optical Coherence Tomography.

TABLE 2: The comparison of ERL-MHSI system and pushbroom MHSI system.

\begin{tabular}{lcc}
\hline Mechanism & $\begin{array}{c}\text { ERL-MHSI } \\
\text { system }\end{array}$ & $\begin{array}{c}\text { Pushbroom MHSI } \\
\text { system }\end{array}$ \\
\hline Volume $\left(\mathrm{cm}^{3}\right)$ & $70(L) \times 55(W)$ & $120(L) \times 100(W)$ \\
$\times 80(H)$ & $\times 90(H)$ \\
Spectral range $(\mathrm{nm})$ & $400-1000$ & $400-1000$ \\
$F / \#$ & 2.8 & 6.5 \\
Spatial resolution $(\mu \mathrm{m})$ & $30 \times 10$ & $20 \times 10$ \\
Spectral resolution $(\mathrm{nm})$ & 2.8 & 7.2 \\
Throughput & $>60 \%$ & $>60 \%$ \\
Off-axis aberration & Good & $\mathrm{Bad}$ \\
Scanning mechanism & Stepping motor & $\mathrm{PZT}$ \\
Stability & Good & $\mathrm{Bad}$ \\
Cost & Low & High \\
\hline
\end{tabular}

spectral information of the cell nucleus was the basis to diagnose the degree of the neoplasia. For the twenty cases, the best sensitivity for identifying normal cells and squamous cell carcinoma (SCC) was $100 \%$. The best specificity for identifying normal cells and SCC was 99\%. The best sensitivity for identifying normal cells and dysplasia was 99\%. The best specificity for identifying normal cells and dysplasia was $97 \%$. This work also applies fractal dimension to analyze the morphological information and find that the value of normal and SCC has big difference.

\section{Materials and Methods}

2.1. Operational Principle of ERL-MHSI System. The section describes the design principle of relay lens and the imaging principle of proposed ERL-MHSI system. The 3D data of hyperspectral image consists of spatial $(x, y)$ and spectral $(\lambda)$ information. The hyperspectral image can be acquired by scanning one axis on the sample. For the microscope application, the hyperspectral image is always acquired by moving the biopsy. We design a scanning relay lens module for HSI in the previous research and now apply it to the MHSI system. The scanning relay lenses module is consisted of RL and SM. In our survey, this is the first time that the relay lens has been applied to MHSI system. The RL resembles a finite conjugate and telecentric system with unity transverse 

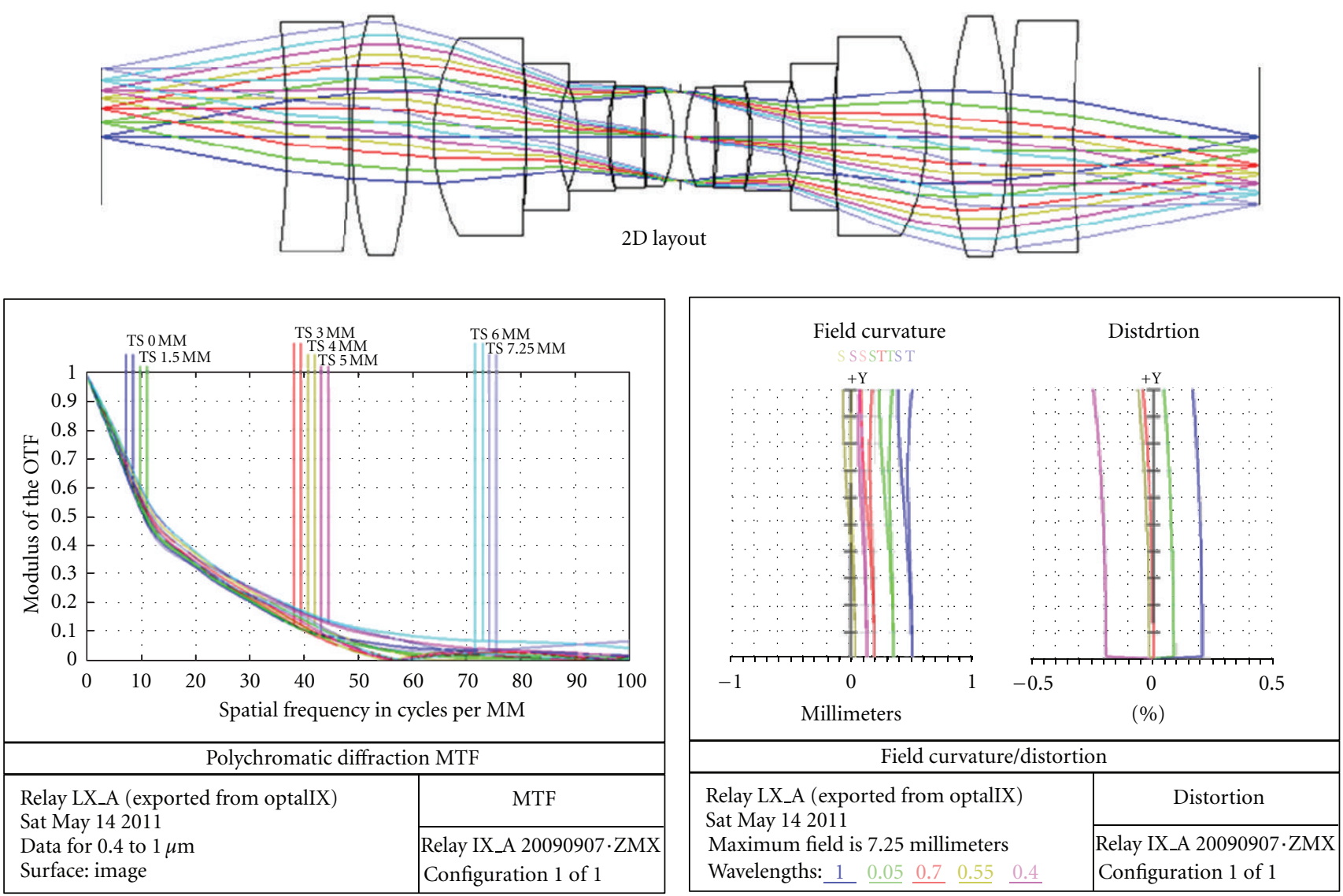

Figure 2: The 2D layout, MTF, and distortion of relay lens.

magnification. A finite conjugate system means that while a light source (not at infinity) passes the lens, it focuses on a particular spot. The designed relay lens consists of two symmetric infinite conjugate lenses with the same focus so as to cancel optical aberration. The telecentricity means that the exit pupil of an optical system is at infinity and the imaging size remains uniform with the variation of focus. Therefore, the off-axis image remains the same as the central image. Besides, even if the focus of light changes, it does not affect the image size and can minimize imaging distortion. Figure 2 shows the 2D layout, MTF, and distortion of RL. The RL consists of 14 lenses. The 5th and 6th lenses are utilized to calibrate color aberration. The size of aperture stop is about $5 \mathrm{~mm}$. The distortion is smaller than $0.02 \%$, total length of relay lens is about $120 \mathrm{~mm}$, magnification is -1 , and the $\mathrm{F} / \#$ is 2.8 .

The ERL-MHSI system provides the transmitting and fluorescence image of biopsy to assist the pathologist to diagnose the grade of cancer. The transmitting image provides the morphological information and the spectral information from $400 \mathrm{~nm}$ to $1000 \mathrm{~nm}$ of the cell or tissue. The diverse cell or tissue absorbs the different spectrum of light. The fluorescence image provides the characteristic spectrum of the cell. Figure 3(a) shows the optical schematic of the ERLMHSI system. The proposed system has two light sources (halogen and xenon). The halogen $(100 \mathrm{~W})$ which locates on the top of the system is used to be the illumination of the transmitting image. The yellow line represents the light path of transmitting image. When the light passes through the biopsy stage (BS), the cell or tissue of the biopsy absorbs the energy which causes that the spectral intensity of some cell or tissue would reduce at its characteristic spectrum. The objective lens (OBL) can form and magnify the image of interested region. In the transmitting mode, the fluorescent mirror unit (FMU) does not open. The beam splitter 1 (BM1) separates the light into two paths. The CCD can immediately capture the biopsy image, and the user can adjust the BS to find the interested region of biopsy. The beam splitter 2 (BM2) guides the light toward to the relay lens (RL). The RL projects the image from imaging plane 1 (IMP1) to imaging plane 2 (IMP2), which is the slit of hyperspectrometer (HM). The IMP1 is the imaging plane of the microscope. The slit with the width of $30 \mu \mathrm{m}$ is located on the $y$-axis and allows for IMP2 image on line at a time on the electron multiplying charge-coupled device (EMCCD). When the RL is static, the slit gets the line image from the IMP2 of circle image. The dispersive structure inner the HM disperse the each point of the line image into spectral axis of the EMCCD. Hence, when the RL is static, the slit image and its spectrum can be record on the EMCCD. And then, the SM moves one step along the $x$-axis to obtain the next line image of slit and its spectrum. While the SM scans along the $x$-axis, 


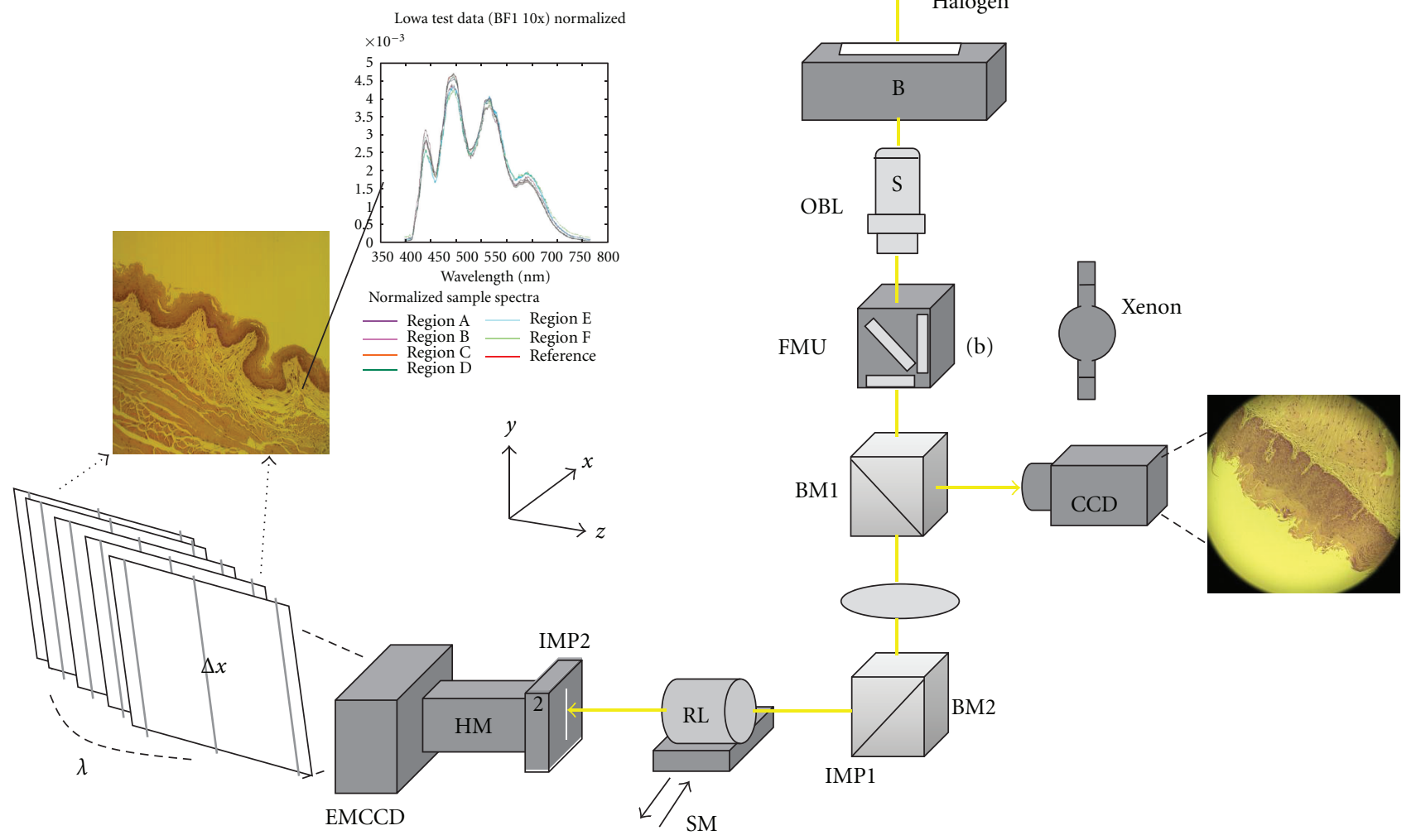

(a)

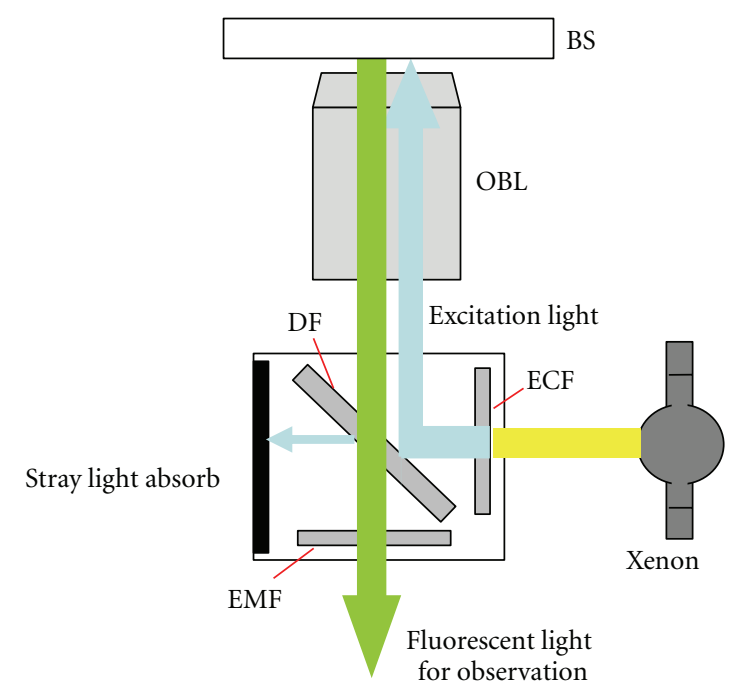

(b)

FIgure 3: (a) The optical schematic of the ERL-MHSI. (b) The optical schematic of fluorescent mirror unit (FMU).

the separate line image is recorded on the $y$ - $\lambda$ plane of the EMCCD. After the line images are all obtained, the data cube of all of the $y$ - $\lambda$ files is loaded to the memory.

In the fluorescent mode, the excitation light source of fluorescent image is xenon lamp which has the wide range spectrum, and the FMU is open. As Figure 3(b) shows, the FMU is composed of excitation filter (ECF), dichromatic mirror (DM), and emission filter (EMF). The excitation band is determined by the ECF. The DM reflects the light to the BS, and then the excitation fluorescent light of the cell or tissue goes back to the DM. However, some stray light of the excitation light transmits without reflection by the DM. The FMU incorporates a mechanism that absorbs more than $99 \%$ of the stray light. The EMF determines the passing spectral 
band of the excitation fluorescent light. And then the light arrives the RL. The following light path and procedure are the same as transmitting mode.

2.2. Analysis and Calibration of ERL-MHSI System. This section describes the hardware specification of the ERL-MHSI system and analyzes the spatial resolution, spectral resolution of the system, and the calibration process before utilization. As Figure 4 shows, the proposed ERL-MHSI system consisted of commercial inverted microscope (Olympus IX71), CCD (AVT PIKE F-421-C), RL, SM (Sigma Koki, SGSP20-20), hyperspectrometer (Specim V10E, with spectral range from 400 to $1000 \mathrm{~nm}$ ), and EMCCD (Andor Luca R604, with $1000 \times 1000$ pixels and $8 \mu \mathrm{m}$ pixel size). The software was written by $\mathrm{C}$ language to connect the hardware for capturing image, analyzing spectral information, and displaying interested region of image by the CCD at the right part of the system. The soft also can control the speed of SM, gain and exposure time of EMCCD. Figure 5 shows the workflow of the ERL-MHSI system.

The spatial resolution of the proposed system was discussed in $x$-axis and $y$-axis, respectively. The spatial resolution of the $x$-axis mainly relates to the entrance slit width of the hyperspectrometer and optical magnification of the entire system. Because the slit width of the proposed system is $30 \mu \mathrm{m}$ and the magnification of the RL is -1 , the spatial resolution of the ERL-MHSI system is $30 \mu \mathrm{m}$. The spatial resolution of $y$-axis is mainly determined by the pixel size of EMCCD, spot size of the relay lens, and magnification of the ERL-MHSI system. Because the pixel size of the EMCCD is $8 \mu \mathrm{m}$ and the spot size of the relay lens is smaller than $10 \mu \mathrm{m}$, the spatial resolution of $y$-axis is about $10 \mu \mathrm{m}$. Hence, the spatial resolution of the ERL-MHSI system is $30 \mu \mathrm{m} \times 10 \mu \mathrm{m}$. However, the objective power directly affects the spatial resolution (for objective power 20x, the spatial resolution is $1.5 \mu \mathrm{m} \times 0.5 \mu \mathrm{m}$ ). The spectral resolution (usually referred as nominal spectral resolution) of the ERL-MHSI system is decided by the capability of dispersing spectrum of the hyperspectrometer, and it is generally determined by the ratio of slit width. For the $80 \mu \mathrm{m}$ slit width, the spectral resolution is about $7.5 \mathrm{~nm}$. Hence, the spectral resolution of the ERL-MHSI system (slit width of $30 \mu \mathrm{m}$ ) is about $2.8 \mathrm{~nm}$.

Before utilization, the proposed system must implement radiometric and spectral calibration. The radiometric calibration is an important task, because the peak quantum efficiency of each pixel on EMCCD is different. A halogen lamp was prepared to be the standard illumination for calibration. Initially, a spectrometer (SphereOptics SMS-500) before utilization was used to measure the standard illumination and then acquired the standard response curve from $400 \mathrm{~nm}$ to $1000 \mathrm{~nm}$. Secondly, a dark image with no illumination to the ERL-MHSI system was utilized to remove the signal noise of the system. Following, a reference slide was used to cancel nonuniformity of the image caused by uneven illumination, periodic scanline strip, the effect of the lamp, medium, and reflectance and transmittance of the biopsy.

The spectral response curve of a standard illumination was distinct from the ERL-MHSI system from 400 to

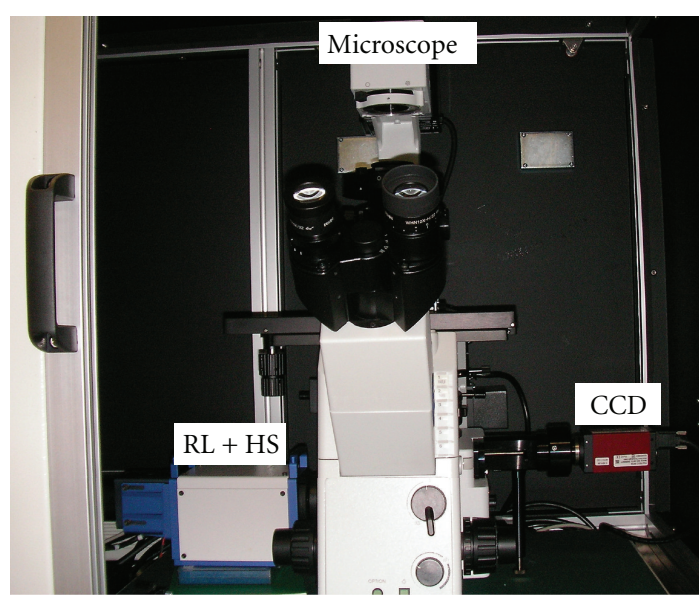

FIGURE 4: The finish product of ERL-MHSI system.

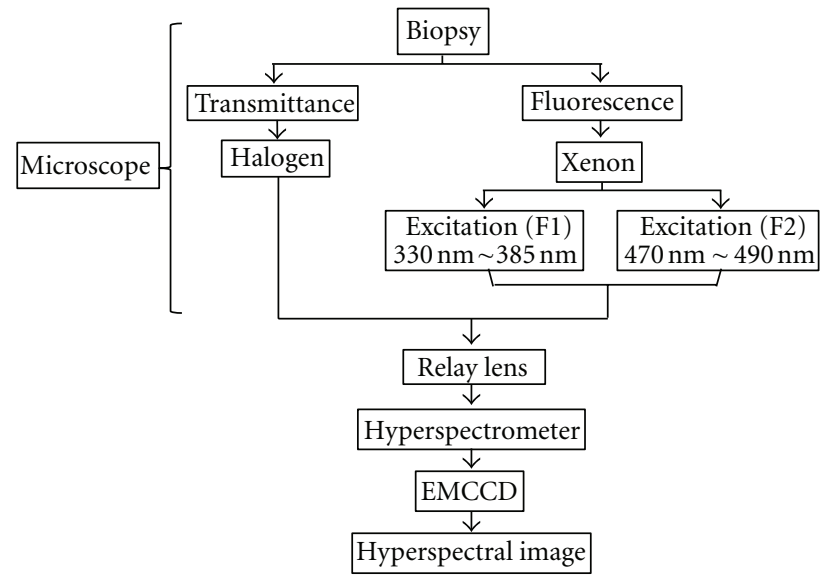

FIgURE 5: The workflow of the ERL-MHSI system.

$1000 \mathrm{~nm}$. The $k$ value was the calibrated parameter, $k=S(\lambda) /$ $H(\lambda)$, where $S(\lambda)$ and $H(\lambda)$, respectively, represent the response value of standard illumination of each wavelength and the response value of the ERL-MHSI system of each wavelength. The spectral calibration guaranteed that all pixels represented the correct wavelength. An Hg-Ar lamp (SphereOptics) was used to be the light source of calibration. The spectrum of the Hg-Ar lamp was, respectively, measured by the spectrometer (SphereOptics SMS-500) and the ERLMHSI system. The measured wavelength of the Hg-Ar lamp was in the same pixel position of these two devices.

2.3. Biopsy Procedure and Data Collection of Mice. This paper was followed to the method of Chang et al. [22] to establish mimicking oral tumorigenesis of twenty mice. The used mouse chow (Prolab RMH 2500 PMI Nutrition International, LLC, MO, USA), 4-NQQ (Sigma-Aldrich, St. Louis, MO, USA), and arecoline hydrobromide (Fluka, Buchs, China) of this experiment were regularly chemical medicines. No authors have any conflict of interest with the three companies. The Six-week-old male C57BL/6JNarl mice were bought from the National Laboratory Animal Center. 
The mice were dealt based on the Animal Care and Use Guidelines of the China Medical University, and the protocol was approved by the Institutional Animal Care Use Committee. These experiments were implemented under controlled conditions of a $12 \mathrm{~h} \mathrm{light/dark}$ cycle. Mice were raised with standard mouse chow (Prolab RMH 2500 PMI Nutrition International, LLC, MO, USA). The carcinogens, $200 \mu \mathrm{m} / \mathrm{mL}$ 4-NQQ (Sigma-Aldrich, St. Louis, MO, USA) and $500 \mu \mathrm{m} / \mathrm{mL}$ arecoline hydrobromide (Fluka, Buchs, China), were dissolved in the drinking water that was replaced once a week. The mice were allowed to access the drinking water and chow diet ad libitum during the treatment. Besides, mice were weighed every 4 weeks. Biweekly, precancerous and cancerous lesions of the tongue were diagnosed and recorded. The mice were exposed to $4-\mathrm{NQO} /$ arecoline for 8 weeks and then observed for additional 20 weeks (28 weeks of total observation). Figure 6(a) was the tongue of lesion of mouse A_11_8 (sample 3). Figure 6(b) was the tongue of lesion of mouse N_2_3 (sample 4). The tongue, lymph nodes, esophagus, spleen, gastrointestinal tract, liver, and kidney were fixed in 10\% formaldehyde. For histopathological diagnosis, paraffin-embedded tongue specimens were stained by hematoxylin and eosin (H\&E). The observed lesions were classified to four types: epithelial hyperplasia, papilloma, dysplasia, and SCC. A macroscopic inspection of other organs, including the esophagus, liver, colon, kidney, spleen, and stomach, was implemented. Specimens were stained with H\&E, and histopathologic diagnosis was used to establish criteria.

This research prepared three biopsies of each mouse. The three biopsies were in the normal, dysplasia, and SCC stages, respectively. After, the pathologist marked the layers of oral tissue, the distribution of cancer cells and normal cells on the biopsies. We used the 20x objective power and two fluorescence illumination (F1: the range of excitation light from $330 \mathrm{~nm}$ to $385 \mathrm{~nm}, \mathrm{~F} 2$ : the range of excitation light from $470 \mathrm{~nm}$ to $490 \mathrm{~nm}$ ) to acquire the MHSI image. The scanning time of each biopsy was about 10 minutes.

2.4. Spectral Data Processing and Analysis. In this research, the analyzed spectral data was from the fluorescence image. Before using the data, the dark field calibration was necessary. The calibration formula is $I_{F}-I_{D}$, where $I_{F}$ represents the spectral intensity of each pixel on the fluorescence image and $I_{D}$ represents the spectral intensity of each pixel in the dark field. Because there were two fluorescence excitation lights of the FMU (F1: $330 \mathrm{~nm}$ to $385 \mathrm{~nm}, \mathrm{~F} 2: 470 \mathrm{~nm}$ to $490 \mathrm{~nm}$ ), two methods were utilized to classify the data. The two methods both based the characteristic of the spectral shape to classify normal cells and cancer cells. We took all cell nucleuses of the fluorescence image. Each cell nucleus was composed of nine pixels. We took about 100 normal cells, 200 dysplasia cells, and 300 SCC from each mouse sample. Equation (1) was the formula of method 1 for F1 results used the peak and valley values to be the characteristic of spectrum. From (1), each cell nucleus can obtain a value. And then the Gaussian distribution was used to statistic these values. From Gaussian distribution, the values were separated

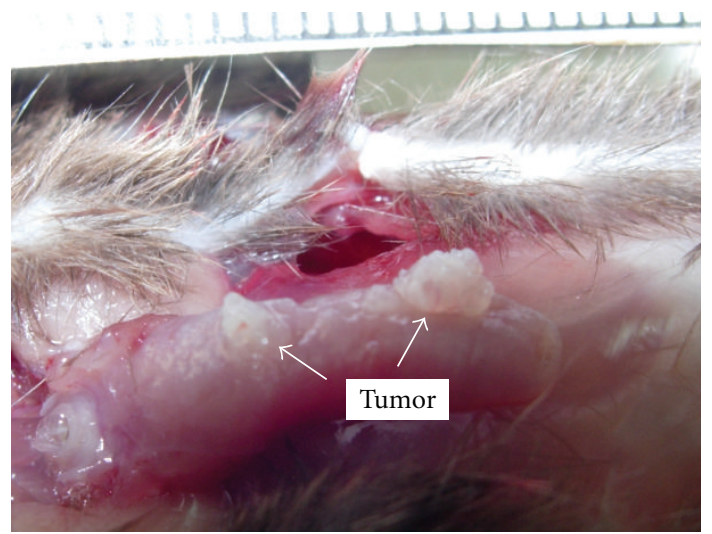

(a)

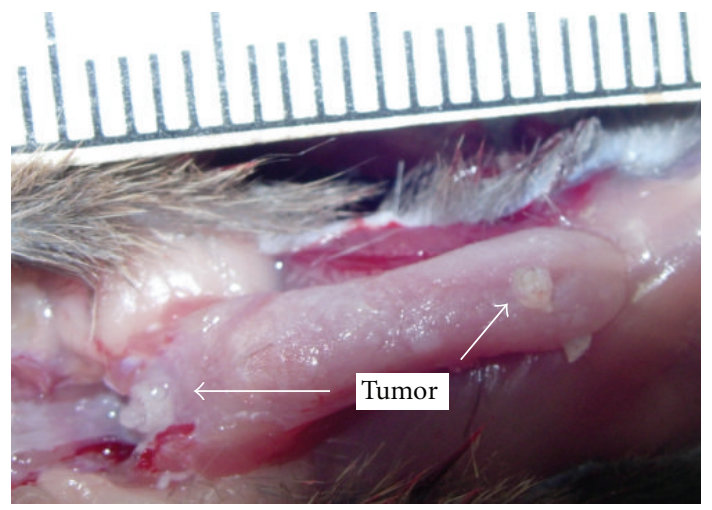

(b)

Figure 6: (a) The tongue of lesion of mouse A_11_8 (sample 3). (b) The tongue of lesion of mouse N_2_3 (sample 4).

into two groups. Finally, the sensitivity for identifying normal cells and dysplasia, normal cells to SCC can be calculated. The method 2 for $\mathrm{F} 2$ results used the difference of the bandwidth among normal, dysplasia, and SCC

$$
\frac{1}{\text { Peak } 1 \times \text { Peak } 2 \times \text { Valley }} \text {. }
$$

2.5. Morphological Data Processing and Analysis. One of the advantages of the ERL-MHSI system is simultaneously to acquire the morphological information from transmitting image and the spectral information of each point. Before analyzing the transmitting image, the pathologist marked the layers of oral epithelial tissue and distribution of cancer cells and normal cells on the transmitting image, and the row data of transmitting image must be calibrated. First, a dark image with no light to ERL-MHSI system was used to remove the dark noise of the system. Second, a reference blank for which an area on the slide was scanned with all layers of glass except the cell structures was used to remove the nonuniformity of the transmitting image caused by the uneven light source, scan line striping, and the effect of lamp, medium, and 
TABLE 3: The spectral characteristic-based identification of twenty mice.

\begin{tabular}{|c|c|c|c|c|c|c|c|c|}
\hline Sample & $\begin{array}{l}\text { F1_N\&S: } \\
\text { sensitivity }\end{array}$ & $\begin{array}{l}\text { F1_N\&S: } \\
\text { specificity }\end{array}$ & $\begin{array}{l}\text { F1_N\&D: } \\
\text { sensitivity }\end{array}$ & $\begin{array}{l}\text { F1_N\&D: } \\
\text { specificity }\end{array}$ & $\begin{array}{l}\text { F2_N\&S: } \\
\text { sensitivity }\end{array}$ & $\begin{array}{l}\text { F2_N\&S: } \\
\text { specificity }\end{array}$ & $\begin{array}{l}\text { F2_N\&D: } \\
\text { sensitivity }\end{array}$ & $\begin{array}{l}\text { F2_N\&D: } \\
\text { specificity }\end{array}$ \\
\hline 1 & 81 & 89 & 72 & 69 & 75 & 78 & 76 & 78 \\
\hline 2 & 93 & 88 & 80 & 92 & 84 & 72 & 74 & 71 \\
\hline 3 & 99 & 97 & 96 & 93 & 86 & 79 & 80 & 72 \\
\hline 4 & 100 & 99 & 99 & 97 & 91 & 90 & 88 & 83 \\
\hline 5 & 90 & 88 & 92 & 85 & 89 & 90 & 89 & 85 \\
\hline 6 & 94 & 90 & 85 & 83 & 78 & 75 & 81 & 68 \\
\hline 7 & 80 & 81 & 83 & 78 & 79 & 81 & 74 & 77 \\
\hline 8 & 93 & 88 & 79 & 77 & 73 & 83 & 73 & 75 \\
\hline 9 & 90 & 89 & 85 & 86 & 82 & 78 & 74 & 61 \\
\hline 10 & 83 & 80 & 64 & 75 & 74 & 88 & 73 & 82 \\
\hline 11 & 83 & 87 & 91 & 80 & 83 & 78 & 83 & 84 \\
\hline 12 & 81 & 66 & 71 & 91 & 74 & 98 & 72 & 90 \\
\hline 13 & 99 & 98 & 81 & 73 & 89 & 88 & 86 & 88 \\
\hline 14 & 92 & 91 & 92 & 90 & 84 & 76 & 93 & 71 \\
\hline 15 & 89 & 75 & 71 & 63 & 81 & 76 & 81 & 79 \\
\hline 16 & 70 & 79 & 63 & 68 & 71 & 67 & 73 & 72 \\
\hline 17 & 85 & 90 & 87 & 86 & 81 & 80 & 77 & 79 \\
\hline 18 & 98 & 96 & 81 & 74 & 83 & 77 & 84 & 72 \\
\hline 19 & 92 & 89 & 81 & 82 & 85 & 83 & 86 & 67 \\
\hline 20 & 85 & 91 & 84 & 82 & 81 & 74 & 68 & 69 \\
\hline AVG. & 88.9 & 87.6 & 81.9 & 81.2 & 81.2 & 80.6 & 79.2 & 76.2 \\
\hline STD. & 7.78 & 8.07 & 9.88 & 9.13 & 5.65 & 7.31 & 6.84 & 7.65 \\
\hline
\end{tabular}

F1 represents the excitation wavelength ranging from $330 \mathrm{~nm}$ to $385 \mathrm{~nm}, \mathrm{~F} 2$ represents $470 \mathrm{~nm} \sim 490 \mathrm{~nm}$. The N represents normal, S represents SCC, and D represents dysplasia. The AVG. represents average value among twenty data and STD. represents standard deviation. The unit of sensitivity and specificity is percentage (\%).

reflectance or transmittance of glass. Equation (2) was the calibration formula

$$
\frac{I_{T}-I_{D}}{I_{B}-I_{D}}
$$

where $I_{T}$ represents the spectral intensity of each pixel on the transmitting image, $I_{D}$ represents the spectral intensity of each pixel in the dark field, and $I_{B}$ represents the spectral intensity of each pixel on the bright field. For discriminating the transmitting image of cancer or normal, this paper used fractal dimension to be the classified value. The fractal dimension was a value which provides a statistics of complexity comparing in a pattern changed with a scale [23]. Equation (3) is the formula of fractal dimension. Because the layers of oral epithelial tissue of normal were in order but of cancer were disordered, the fractal dimension of the normal and cancer tissue may obviously be different which can help the pathologist to more easily discriminate them

$$
D=\frac{\log N}{\log s},
$$

where $D$ is the fractal dimension, $s$ represents the length of the chose smallest unit, and $N$ represents the number of $s$ to cover the pattern.

\section{Results and Discussions}

3.1. Spectral Characteristic-Based and Morphological Identification of Mouse A_11_8. In order to prove that the proposed system was suit to apply to diagnose oral cancer. This paper used twenty mice to be test samples. Tables 3 and 4 list the spectral and morphological results, respectively. This section shows and discusses two best cases of the twenty mice. Figure 7 shows the biopsy image of A_11_8 mouse (sample 3). The ERL-MHSI system has the capable of producing good quality. From the transmitting images ((a), (d), and (g)), the cell of SCC was obvious more than normal or dysplasia. This was because the neoplasia represents the cells abnormal increase. Hence, the analytic data of SCC was more than dysplasia and normal tissue. The total analytic data of the mouse were 700 cells (normal: 100 cells, dysplasia: 200 cells, and SCC: 400 cells). One data represented one cell nucleus which is represented by nine pixels. Figure 8(a) shows the average fluorescence spectral characteristic of normal, dysplasia, and SCC under F1 illumination. The result showed that these three spectral shapes had the same peak on $550 \mathrm{~nm}$ and $700 \mathrm{~nm}$. The valley was on the $630 \mathrm{~nm}$. Besides, the dysplasia cell had another peak about on the $530 \mathrm{~nm}$ and the normal cell had the lowest intensity. We used the peak and valleys to be the characteristic of the spectral 


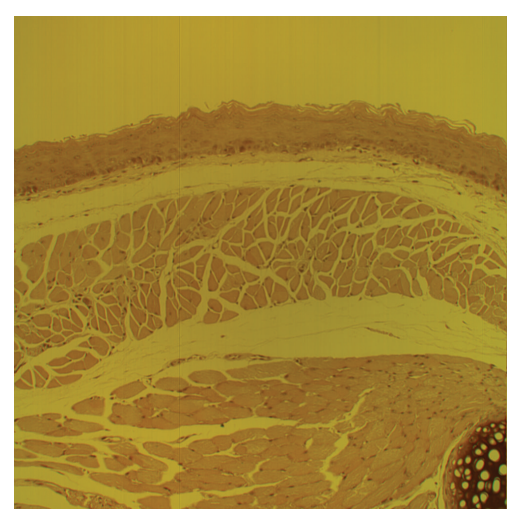

(a)

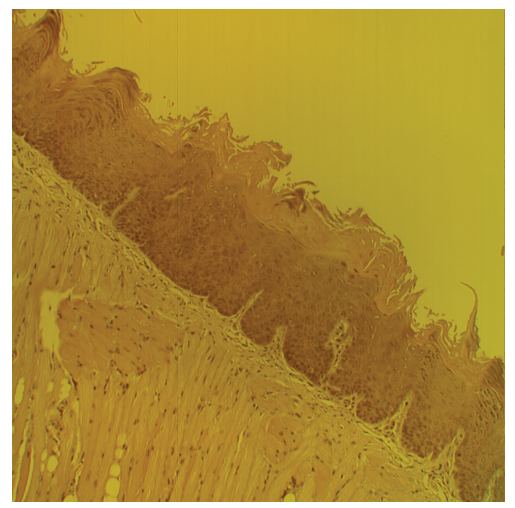

(d)

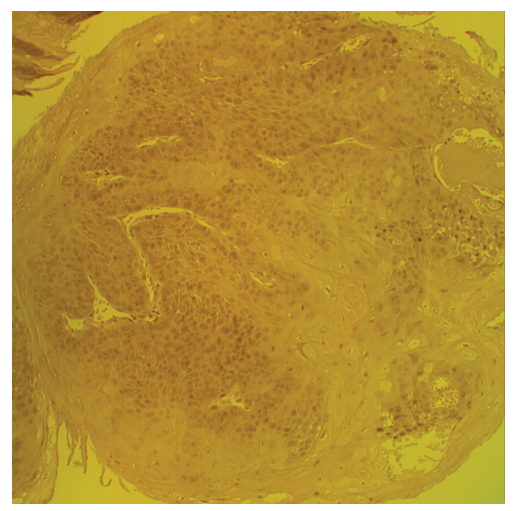

(g)

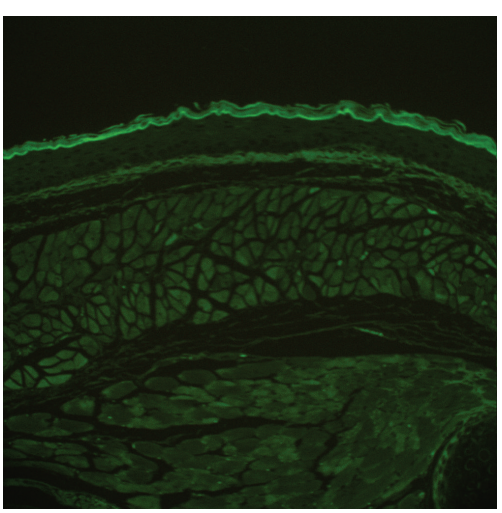

(b)

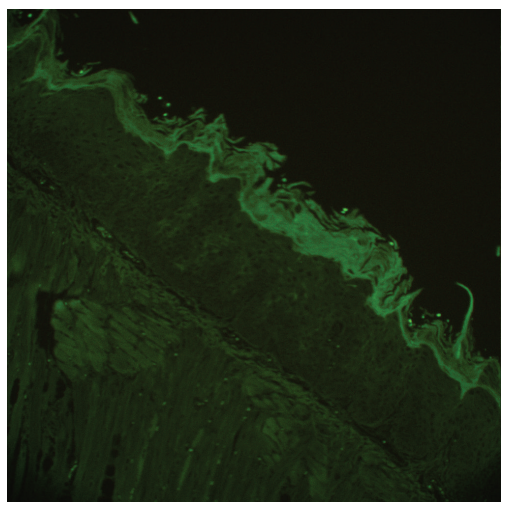

(e)

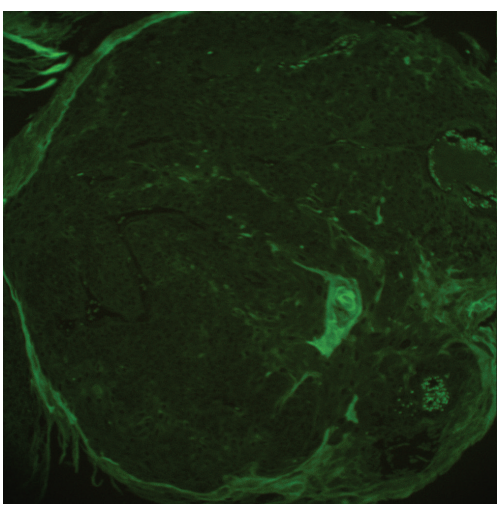

(h)

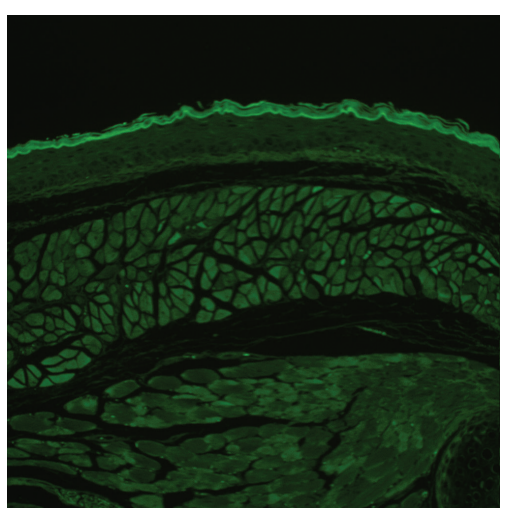

(c)

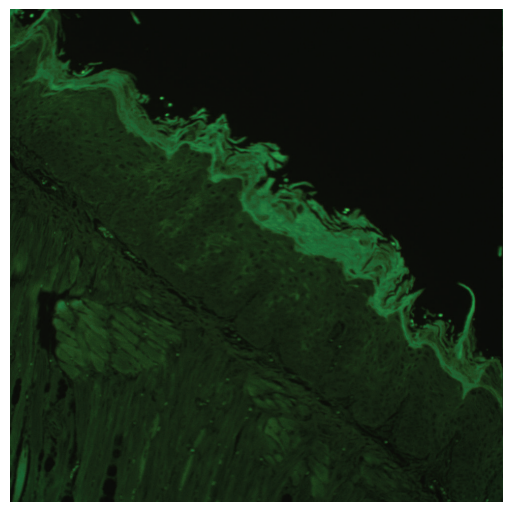

(f)

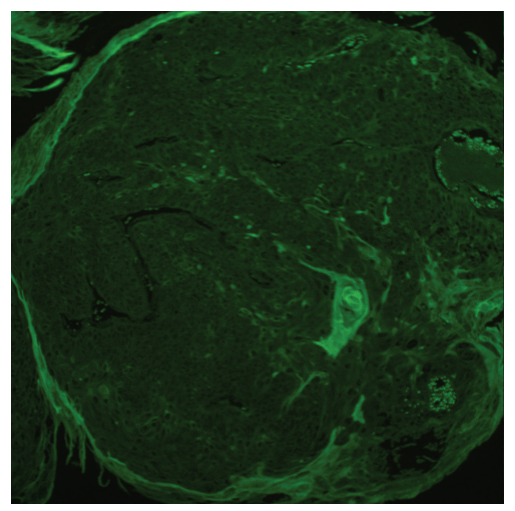

(i)

FIgURE 7: The biopsy image of mouse A_11_8 (sample 3). (a) The transmitting image of normal tissue. (b) The F1 excitation image of normal tissue. (c) The F2 excitation image of normal tissue. (d) The transmitting image of dysplasia. (e) The F1 excitation image of dysplasia. (f) The F2 excitation image of dysplasia. (g) The transmitting image of SCC. (h) The F1 excitation image of SCC. (i) The F2 excitation image of SCC.

shape and then calculated a value of each cell. The sensitivity for identifying normal cells and SCC was 99\%. The specificity for identifying normal cells and SCC was $97 \%$. The sensitivity for identifying normal cells and dysplasia was $96 \%$. The specificity for identifying normal cells and dysplasia was $93 \%$. Figure 8(b) shows the average fluorescence spectral characteristic of normal, dysplasia, and SCC under F2 illumination. The result showed that, the band width of these three spectral shapes was different. We calculated the band width of each cell and obtained a value. And then the Gaussian distribution was used to separate 700 values into two groups. The sensitivity for identifying normal cells and SCC was $86 \%$. The specificity for identifying normal cells and SCC was $79 \%$. The sensitivity for identifying normal cells and dysplasia was $80 \%$. The specificity for identifying normal cells and dysplasia was $72 \%$. 


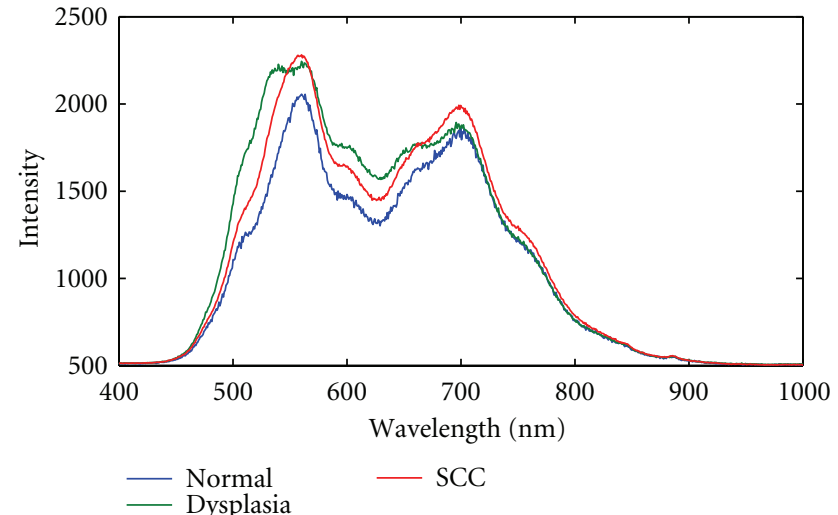

(a)

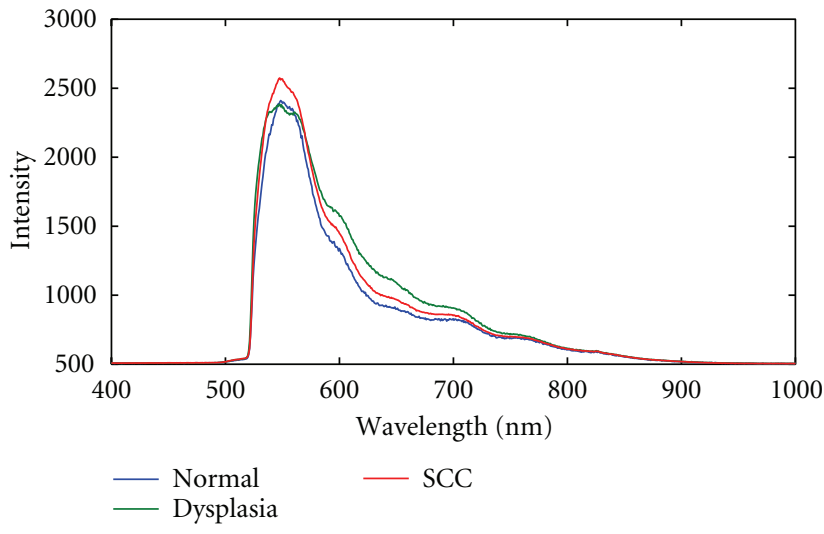

(b)

FIGURE 8: The fluorescence spectral data of normal cells, dysplasia, and SCC of A_11_8 mouse (sample 3). (a) The results by F1 excitation illumination. (b) The results by F2 excitation illumination.

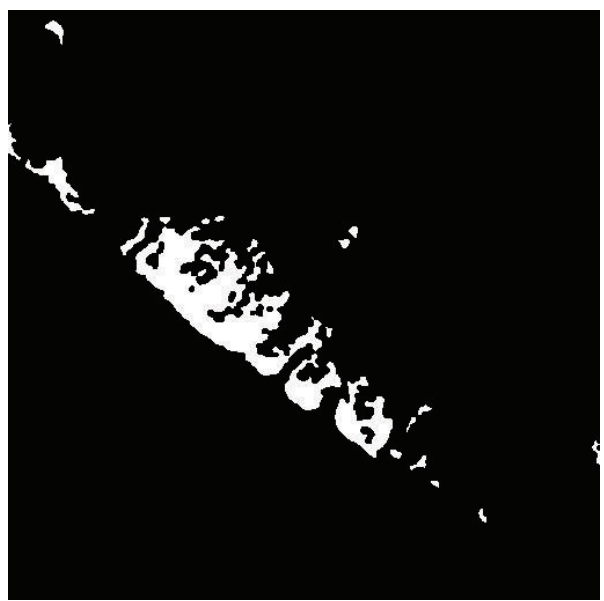

(a)

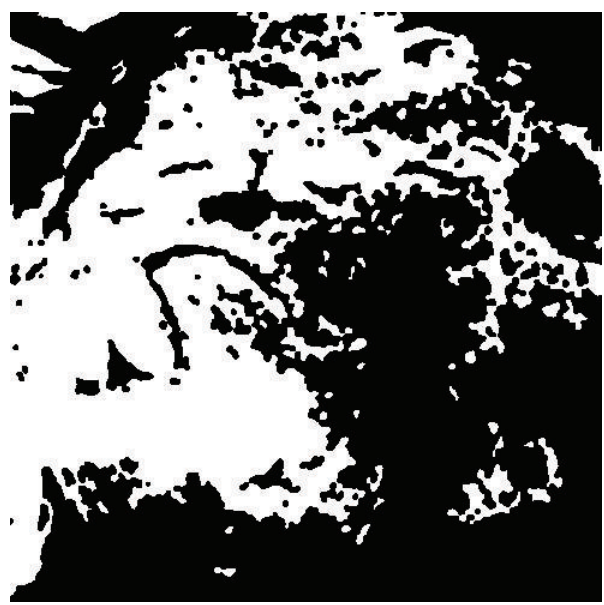

(b)

FIGURE 9: The fractal dimension pattern of dysplasia and SCC of A_11_8 mouse (sample 3). (a) Dysplasia. (b) SCC.

Figure 9 shows the pattern of fractal dimension. Because the fractal dimension of normal tissue and dysplasia was very close, Figure 9 only shows the pattern of dysplasia and SCC. In order to calculate the fractal dimension value, the data of the pattern was preprocessed by binarization and fuzzifierion. The black part of the pattern represents the cell nuclei distribution. The pattern between dysplasia and SCC has significant difference. The value of fractal dimension of normal, dysplasia, and SCC was 1.53, 1.73, and 1.88, respectively. This was because the SCC was more disorder than the dysplasia.

\subsection{Spectral Characteristic-Based and Morphological Identifi-} cation of Mouse N_2_3. Figure 10 shows the biopsy image of N_2_3 (sample 4) mouse. The total analytic data of the mouse were 730 cells (normal: 150 cells, dysplasia: 250 cells, and SCC: 330 cells). Figure 11(a) shows the average fluorescence spectral characteristic of normal, dysplasia, and SCC under
F1 illumination. These three spectral shapes had the same peak on $550 \mathrm{~nm}$ and $700 \mathrm{~nm}$, and the valley was on the $630 \mathrm{~nm}$. The same with A_11_8 mouse, the normal cell had the lowest intensity. However, the $530 \mathrm{~nm}$ peak was not obvious in the mouse. The sensitivity for identifying normal cells and SCC was $100 \%$. The specificity for identifying normal cells and SCC was 99\%. The sensitivity for identifying normal cells and dysplasia was $99 \%$. The specificity for identifying normal cells and dysplasia was $97 \%$. Figure 11 (b) shows the average fluorescence spectral characteristic of normal, dysplasia, and SCC under F2 illumination. The band width of these three spectral shapes was different. The sensitivity for identifying normal cells and SCC was $91 \%$. The specificity for identifying normal cells and SCC was $90 \%$. The sensitivity for identifying normal cells and dysplasia was $88 \%$. The specificity for identifying normal cells and dysplasia was $83 \%$. Figure 12 shows the pattern of fractal dimension. The value of fractal dimension of normal, dysplasia, and SCC was $1.62,1.69$, and 1.85 , respectively. 


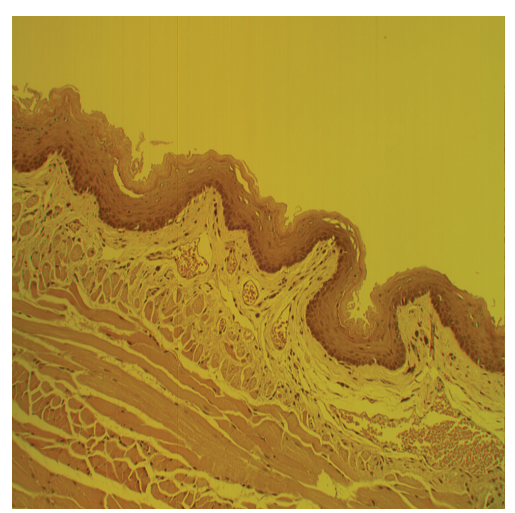

(a)

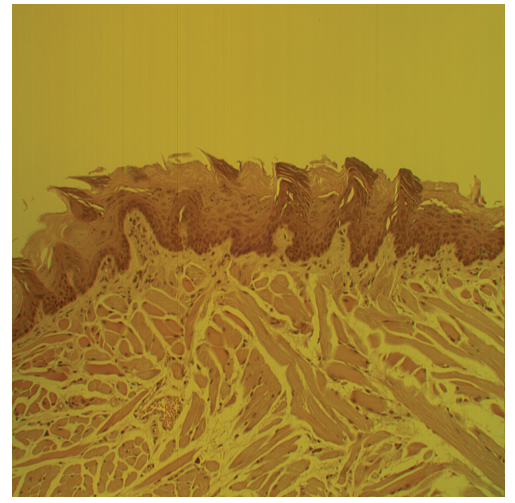

(d)

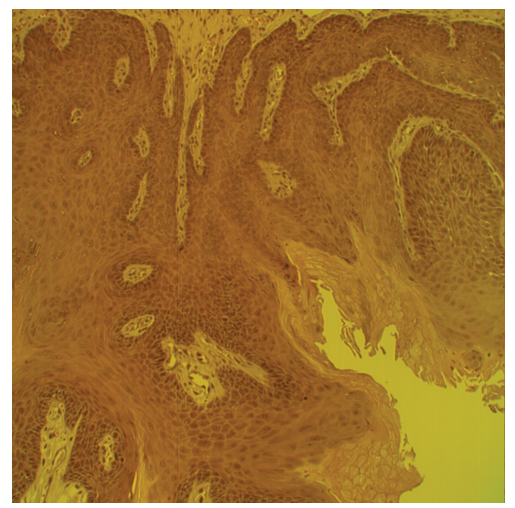

(g)

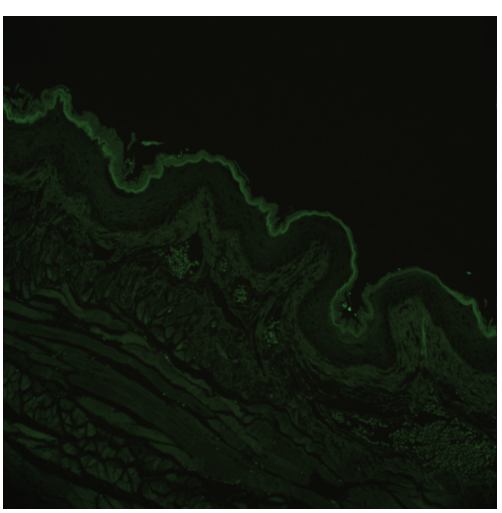

(b)

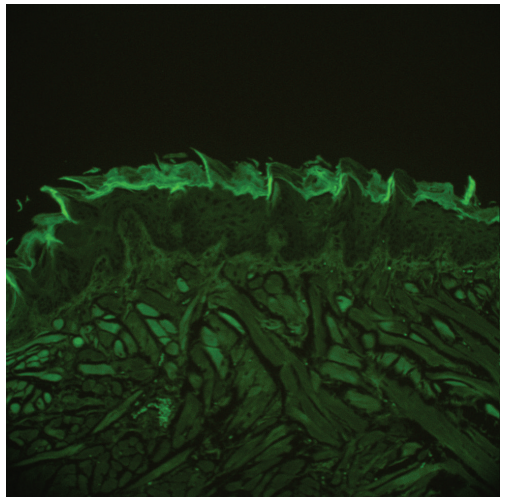

(e)

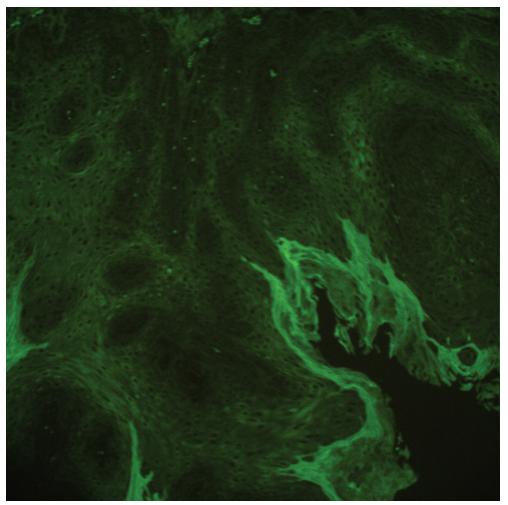

(h)

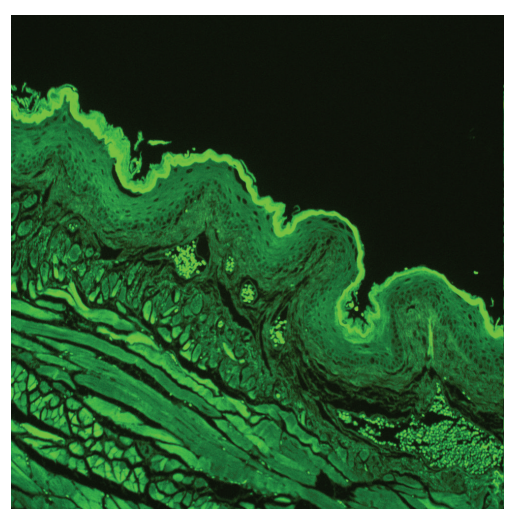

(c)

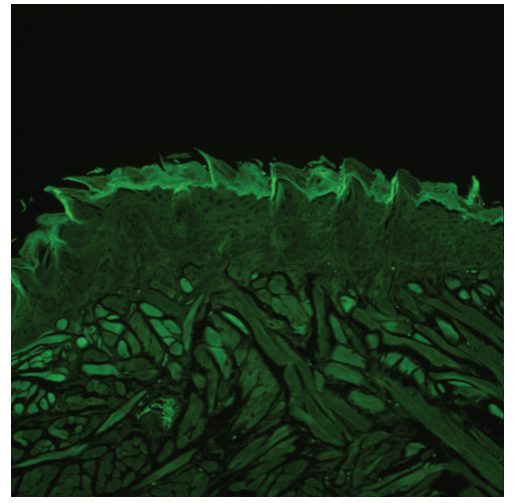

(f)

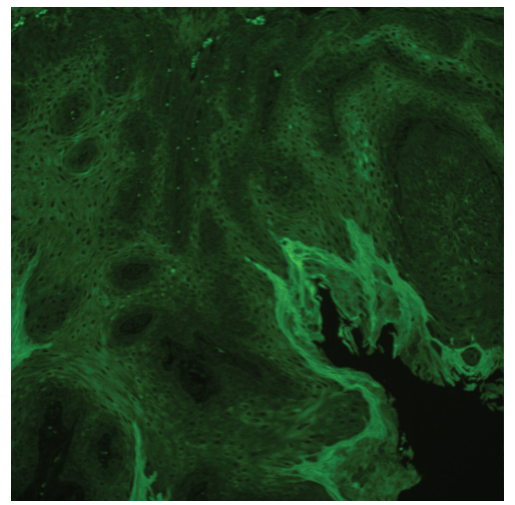

(i)

Figure 10: The biopsy image of mouse N_2_3 (sample 4). (a) The transmitting image of normal tissue. (b) The F1 excitation image of normal tissue. (c) The F2 excitation image of normal tissue. (d) The transmitting image of dysplasia. (e) The F1 excitation image of dysplasia. (f) The F2 excitation image of dysplasia. (g) The transmitting image of SCC. (h) The F1 excitation image of SCC. (i) The F2 excitation image of SCC.

\section{Conclusions}

The novel ERL-MHSI system for cancer diagnosis has been successfully developed in this work. The proposed system has the advantages of simple mechanical and optical structure, easy alignment, stable scanning image quality, good off-axis optical aberration, and lower cost over the conventional MHSI system. Because the ERL-MHSI data can provide both spatial characters and spectral signatures of biopsy, the information can potentially utilize for identification of pathological changes based on particular biochemical and structural features. In order to demonstrate the capability, this study applies the proposed system to diagnose early stage oral cancer of mice. Under F1 illumination (excitation wavelength ranging from $330 \mathrm{~nm}$ to $385 \mathrm{~nm}$ ), the fluorescence spectrum of normal, dysplasia, and SCC has the common feature with two peaks on $550 \mathrm{~nm}$ and $700 \mathrm{~nm}$ and valley on $630 \mathrm{~nm}$. Besides, there is one obvious peak on $530 \mathrm{~nm}$ of dysplasia, and normal cell always has the lowest spectral intensity. Table 3 lists the identification of the fluorescence 


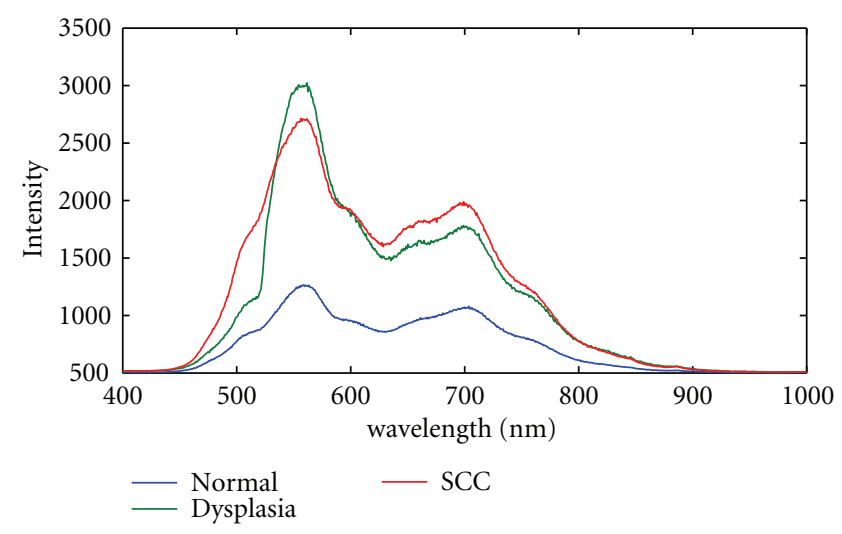

(a)

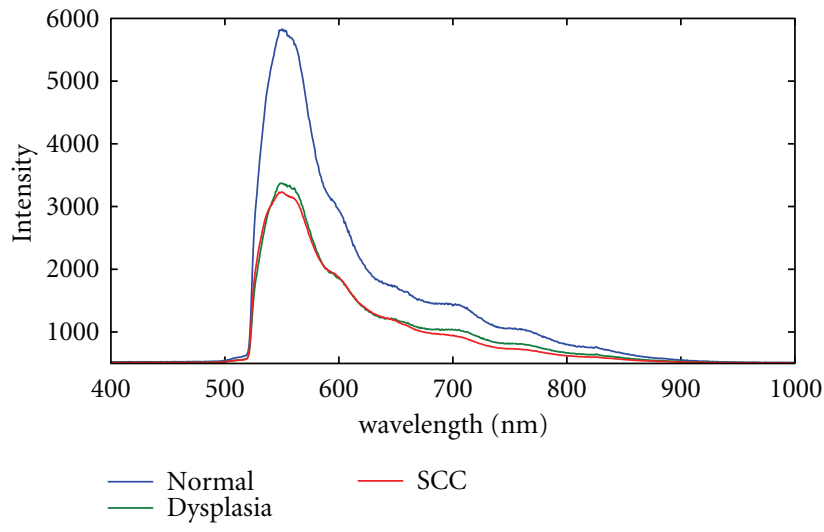

(b)

Figure 11: The fluorescence spectral data of normal cells, dysplasia, and SCC of N_2_3 mouse (sample 4). (a) The results by F1 excitation illumination. (b) The results by F2 excitation illumination.

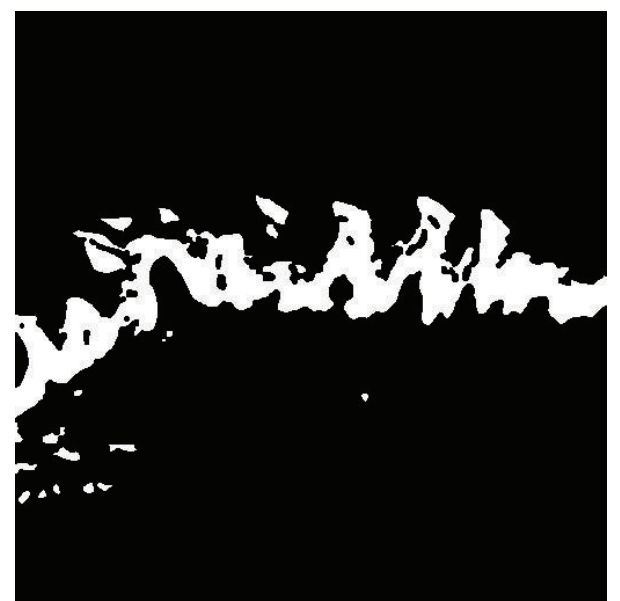

(a)

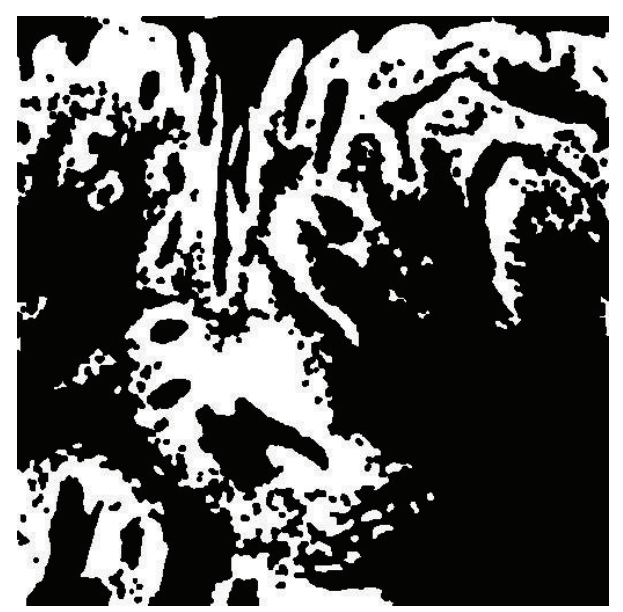

(b)

FIGURE 12: The fractal dimension pattern of dysplasia and SCC of N_2_3 mouse (sample 4). (a) Dysplasia. (b) SCC.
TABle 4: The fractal dimension value for normal, dysplasia, and SCC of twenty mice.

\begin{tabular}{|c|c|c|c|}
\hline Sample & Normal & Dysplasia & SCC \\
\hline 1 & 1.41 & 1.66 & 1.91 \\
\hline 2 & 1.5 & 1.698 & 1.87 \\
\hline 3 & 1.53 & 1.73 & 1.88 \\
\hline 4 & 1.62 & 1.69 & 1.85 \\
\hline 5 & 1.503 & 1.72 & 1.887 \\
\hline 6 & 1.45 & 1.77 & 1.913 \\
\hline 7 & 1.55 & 1.68 & 1.85 \\
\hline 8 & 1.66 & 1.72 & 1.93 \\
\hline 9 & 1.56 & 1.78 & 1.912 \\
\hline 10 & 1.55 & 1.62 & 1.892 \\
\hline 11 & 1.61 & 1.65 & 1.92 \\
\hline 12 & 1.5 & 1.74 & 1.878 \\
\hline 13 & 1.59 & 1.67 & 1.923 \\
\hline 14 & 1.53 & 1.66 & 1.89 \\
\hline 15 & 1.63 & 1.69 & 1.99 \\
\hline 16 & 1.653 & 1.72 & 1.872 \\
\hline 17 & 1.51 & 1.692 & 1.921 \\
\hline 18 & 1.51 & 1.65 & 1.883 \\
\hline 19 & 1.49 & 1.65 & 1.913 \\
\hline 20 & 1.55 & 1.7 & 1.898 \\
\hline AVG. & 1.545 & 1.695 & 1.899 \\
\hline STD. & 0.0662 & 0.0417 & 0.0309 \\
\hline
\end{tabular}

The AVG. represents average value among twenty data and STD. represents standard deviation.

spectral feature among normal, dysplasia, and SCC. The identification of F1 is excellent, but the F2 excitation is undesirable. Hence, the range of excitation wavelength from $330 \mathrm{~nm}$ to $385 \mathrm{~nm}$ is better for diagnosing oral cancer. Besides, the identification between normal and dysplasia is good (the average sensitivity and specificity are resp., $81.9 \%$ 
and $81.2 \%$ ). Therefore, the early stage oral cancer diagnosis is successful. This study also analyzes the morphological information by fractal dimension. The fractal dimension represents the distribution of cell nucleus and the layer of oral tissue. The lower fractal dimension means that the cell nuclei are in order, and the layer of oral tissue is obvious. Table 4 lists the results of fractal dimension. The fractal dimension has significant difference between the normal tissue (about 1.55) and the SCC (about 1.9). However, the difference of fractal dimension for normal and dysplasia is not significant. This is because the structure of the normal and dysplasia tissues has no eventful difference. In the future, we will analyze more biopsies to find the criterion of the fractal dimension. Furthermore, we are ongoing analyzing the human oral cancer biopsy. We are establishing a database of the human oral cancer. Ultimately, the in vivo diagnosis instrument for oral cancer will be developed.

\section{Acknowledgments}

This paper is particularly supported by "Aim for the Top University Plan" of the National Chiao Tung University, Ministry of Education of Taiwan, China Medical University, and National Science Council of Taiwan (Contract no. NSC 101-2220-E-009-032 and NSC 100-2218-E-039-001). The authors want to thank them for providing experimental assistance and related information.

\section{References}

[1] C. T. Willoughby, M. A. Folkman, and M. A. Figueroa, "Application of hyperspectral imaging spectrometer systems to industrial inspection," in Three-Dimensional and Unconventional Imaging for Industrial Inspection and Metrology, vol. 2599 of Proceedings of SPIE, pp. 264-272, October 1995.

[2] B. Khoobehi, J. M. Beach, and H. Kawano, "Hyperspectral imaging for measurement of oxygen saturation in the optic nerve head," Investigative Ophthalmology and Visual Science, vol. 45, no. 5, pp. 1464-1472, 2004.

[3] W. R. Johnson, D. W. Wilson, W. Fink, M. Humayun, and G. Bearman, "Snapshot hyperspectral imaging in ophthalmology," Journal of Biomedical Optics, vol. 12, no. 1, Article ID 014036, 2007.

[4] B. Farina, C. Bartoli, A. Bono et al., "Multispectral imaging approach in the diagnosis of cutaneous melanoma: potentiality and limits," Physics in Medicine and Biology, vol. 45, no. 5, pp. 1243-1254, 2000.

[5] L. L. Randeberg, I. Baarstad, T. Løke, P. Kaspersen, and L. O. Svaasand, "Hyperspectral imaging of bruised skin," in Photonic Therapeutics and Diagnostics II, vol. 6078 of Proceedings of SPIE, p. 60780O, January 2006.

[6] G. N. Stamatas and N. Kollias, "In vivo documentation of cutaneous inflammation using spectral imaging," Journal of Biomedical Optics, vol. 12, no. 5, Article ID 051603, 2007.

[7] A. Vogel, V. V. Chernomordik, J. D. Riley et al., "Using noninvasive multispectral imaging to quantitatively assess tissue vasculature," Journal of Biomedical Optics, vol. 12, no. 5, Article ID 051604, 2007.
[8] D. G. Ferris, R. A. Lawhead, E. D. Dickman et al., "Multimodal hyperspectral imaging for the noninvasive diagnosis of cervical neoplasia," Journal of Lower Genital Tract Disease, vol. 5, no. 2, pp. 65-72, 2001.

[9] M. E. Martin, M. B. Wabuyele, K. Chen et al., "Development of an advanced hyperspectral imaging (HSI) system with applications for cancer detection," Annals of Biomedical Engineering, vol. 34, no. 6, pp. 1061-1068, 2006.

[10] B. S. Sorg, M. E. Hardee, N. Agarwal, B. J. Moeller, and M. W. Dewhirst, "Spectral imaging facilitates visualization and measurements of unstable and abnormal microvascular oxygen transport in tumors," Journal of Biomedical Optics, vol. 13, no. 1, Article ID 014026, 2008.

[11] R. Schultz, T. Nielsen, J. Zavaleta, R. Ruch, R. Wyatt, and H. Garner, "Hyperspectral imaging: a novel approach for microscopic analysis," Cytometry, vol. 43, no. 4, pp. 239-247, 2001.

[12] K. Onizawa, H. Saginoya, Y. Furuya, and H. Yoshida, "Fluorescence photography as a diagnostic method for oral cancer," Cancer Letters, vol. 108, no. 1, pp. 61-66, 1996.

[13] G. J. Tearney, R. H. Webb, and B. E. Bouma, "Spectrally encoded confocal microscopy," Optics Letters, vol. 23, no. 15, pp. 1152-1154, 1998.

[14] S. H. Yun, G. J. Tearney, J. F. de Boer, N. Iftimia, and B. E. Bouma, "High-speed optical frequency-domain imaging," Optics Express, vol. 11, no. 22, pp. 2953-2963, 2003.

[15] D. Yelin, C. Boudoux, B. E. Bouma, and G. J. Tearney, "Large area confocal microscopy," Optics Letters, vol. 32, no. 9, pp. 1102-1104, 2007.

[16] A. M. Siddiqi, H. Li, F. Faruque et al., "Use of hyperspectral imaging to distinguish normal, precancerous, and cancerous cells," Cancer Cytopathology, vol. 114, no. 1, pp. 13-21, 2008.

[17] M. E. Martin, M. B. Wabuyele, M. Panjehpour, M. N. Phan, B. F. Overholt, and T. V. Dinh, "Hyperspectral fluorescence imaging system for biomedical diagnostics," in Advanced Biomedical and Clinical Diagnostic Systems IV, vol. 6080 of Proceedings of SPIE, p. 60800Q, January 2006.

[18] H. Akbari, K. Uto, Y. Kosugi, K. Kojima, and N. Tanaka, "Cancer detection using infrared hyperspectral imaging," Cancer Science, vol. 102, no. 4, pp. 852-857, 2011.

[19] K. Masood, N. Rajpoot, K. Rajpoot, and H. Qureshi, "Hyperspectral colon tissue classification using morphological analysis," in Proceedings of the 2nd Annual International Conference on Emerging Techonologies (ICET '06), pp. 735-741, Peshawar, Pakistan, November 2006.

[20] Y. F. Hsieh, M. Ou-Yang, and C. C. Lee, "Finite conjugate embedded relay lens hyperspectral imaging system," Applied Optics, vol. 50, no. 33, pp. 6198-66205, 2011.

[21] American Cancer Society, Cancer facts \& figures 2012, http:// www.cancer.org/acs/groups/content/@epidemiologysurveilance/documents/document/acspc-031941.pdf.

[22] N. W. Chang, R. J. Pei, H. C. Tseng et al., "Co-treating with arecoline and 4-nitroquinoline 1-oxide to establish a mouse model mimicking oral tumorigenesis," Chemico-Biological Interactions, vol. 183, no. 1, pp. 231-237, 2010.

[23] F. Kenneth, Fractal Geometry, John Wiley \& Sons, 2003. 


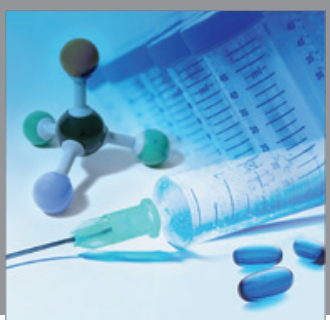

International Journal of

Medicinal Chemistry

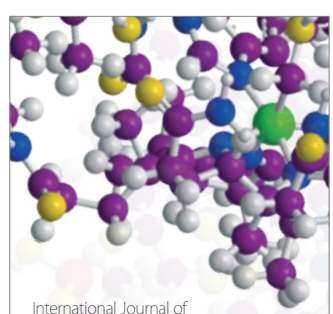

Carbohydrate Chemistry

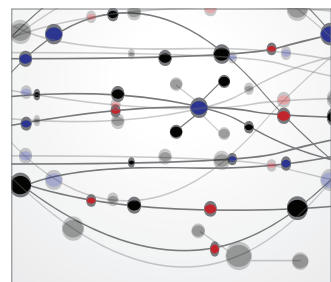

The Scientific World Journal
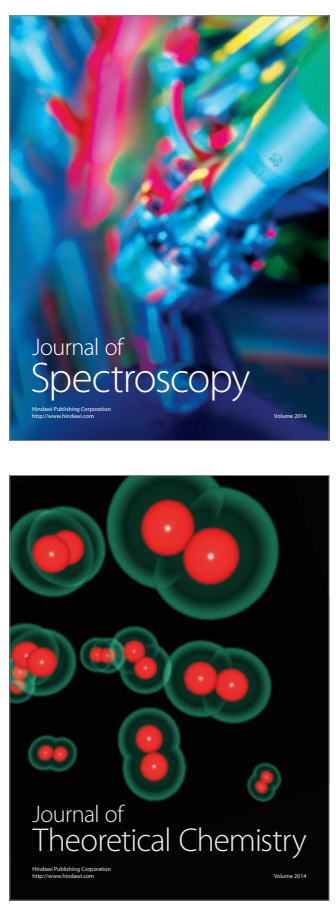
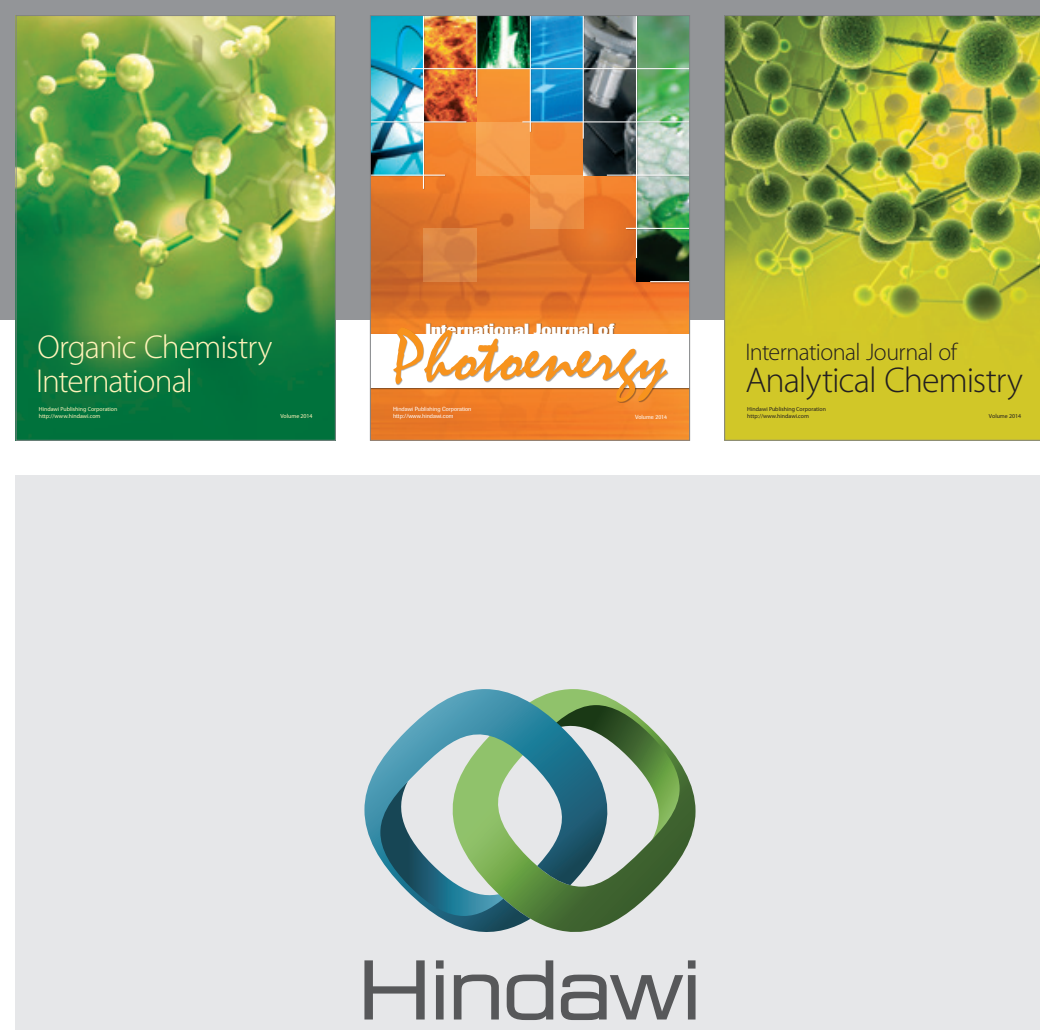

Submit your manuscripts at

http://www.hindawi.com
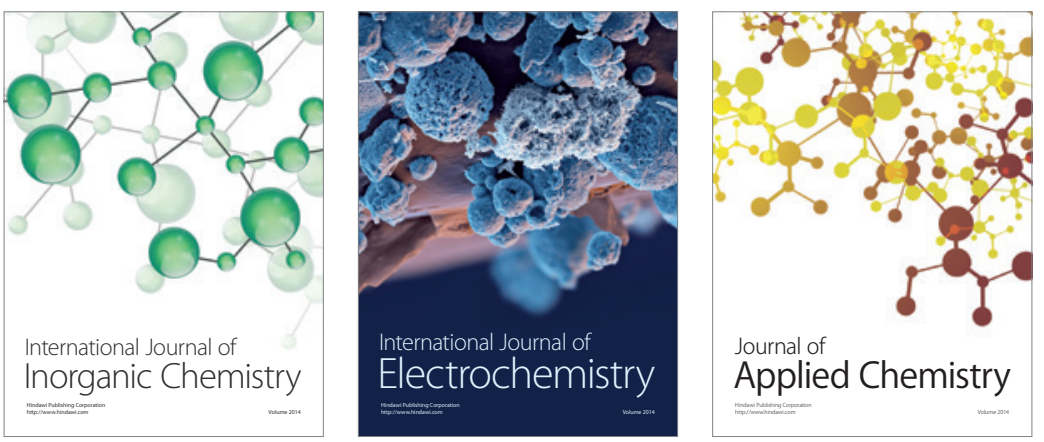

Journal of

Applied Chemistry
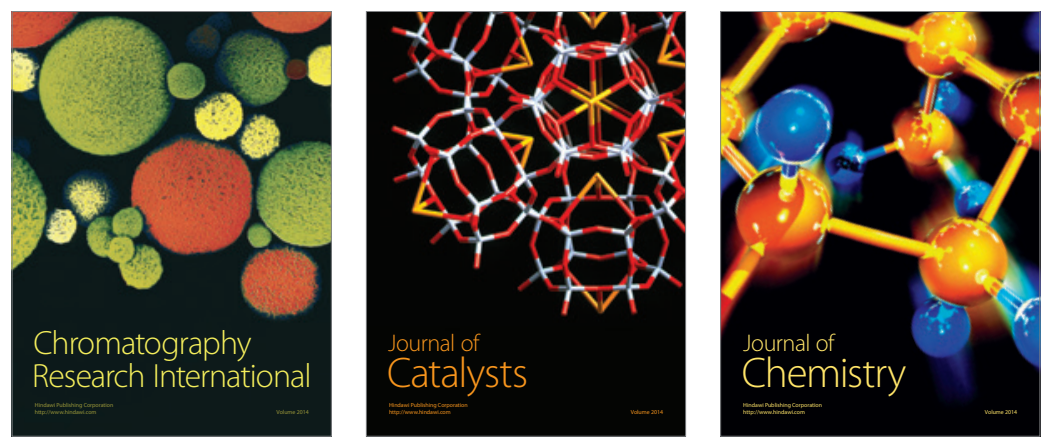
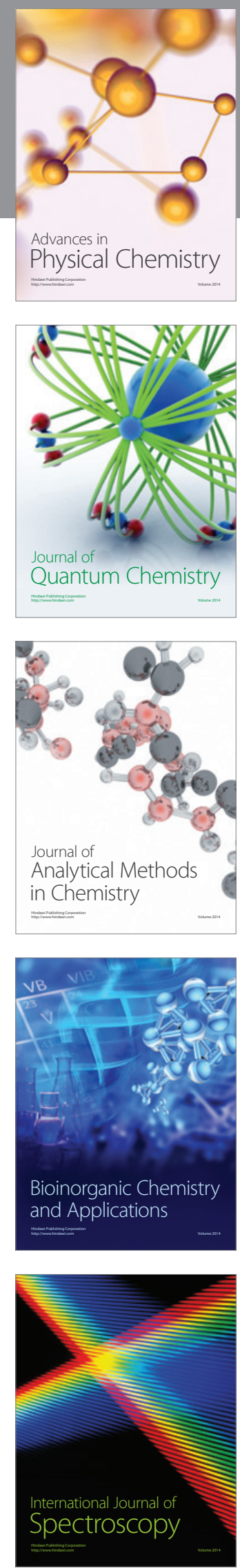